\title{
A CHARACTERISATION OF RIESZ PROXIMITIES
}

\author{
K. C. CHATTOPADHYAY AND H. L. VASUDEVA
}

Abstract. The purpose of this note is to characterise separated Riesz proximities generated by clusters.

1. Introduction. In the theory of proximity spaces of Efremovič [2], Smirnov [5] proved the following result.

A set $X$ with a binary relation ' $A$ close to $B$ ' written $(A \Pi B)$ is a proximity space iff there exists a compact Hausdorff space $Y$ in which $X$ can be topologically embedded so that

( $\bar{A}$ denotes the closure of $A$ ).

$A \Pi B$ in $X$ iff $\bar{A} \cap \bar{B} \neq \varnothing$,

The above result characterises Efremovič proximities. Lodato [3] characterised what are now known as Lodato proximities. The purpose of this note is to characterise Riesz proximities.

\section{Preliminaries.}

2.1. Definitions. Let $X$ be a set, and $c: P(X) \rightarrow P(X)$ a map with the properties: $c(\varnothing)=\varnothing, A \subset c(A)$ for each $A$ in $P(X)$ and $c(A \cup B)=c(A)$ $\cup c(B)$ for $A, B$ in $P(X)$. Then $c$ is called a Čech closure operator and the pair $(X, c)$ is called a Čech closure space. A closure space $(X, c)$ is $R_{0}$ if for any two points $x$ and $y$ of $X, x \in c(y)$ implies $y \in c(x)$. It is called $R_{1}$ if for any $x$ in $X$ and $A$ in $P(X), c(x) \cap c(A) \neq \varnothing$ implies $x \in c(A)$.

Let $(X, c)$ be a closure space and $Y \subset X$. Define $c_{Y}: P(Y) \rightarrow P(Y)$ by $c_{Y}(A)=c(A) \cap Y$ for $A \in P(Y)$. It is easy to verify that $c_{Y}$ is a closure operator on $Y$. The pair $\left(Y, c_{Y}\right)$ is called a subspace of $(X, c)$. A mapping $f$ of the closure space $\left(Y_{1}, c_{1}\right)$ into the closure space $\left(Y_{2}, c_{2}\right)$ is said to be cl-continuous if $f\left(c_{1}(A)\right) \subset c_{2}(f(A)) \forall A \in P\left(Y_{1}\right)$. A one-one mapping $f$ of the closure space $\left(Y_{1}, c_{1}\right)$ onto the closure space $\left(Y_{2}, c_{2}\right)$ is said to be a $c l$-isomorphism of $\left(Y_{1}, c_{1}\right)$ onto $\left(Y_{2}, c_{2}\right)$ if both $f$ and $f^{-1}$ are cl-continuous.

2.2. Riesz proximity spaces. As in Thron [4] we define a basic proximity space to be an abstract set $X$ with a binary relation $\Pi$ on its power set satisfying the following axioms: (i) $\Pi=\Pi^{-1}$, (ii) $A \cup B \in \Pi(C)$ iff $A \in$ $\Pi(C)$ or $B \in \Pi(C)$, (iii) $A \cap B \neq \varnothing$ implies $A \in \Pi(B)$, (iv) $\varnothing \notin \Pi(A)$ for every $A \in P(X)$.

Here $\Pi(A)=[B:(B, A) \in \Pi]$. When $\Pi$ is a basic proximity on $X$, then the pair $(X, \Pi)$ is called a basic proximity space. A proximity space $(X, \Pi)$ is said

Received by the editors March 9, 1976 and, in revised form, August 20, 1976.

AMS (MOS) subject classifications (1970). Primary 54E05.

(c) American Mathematical Society 1977 
to be separated if $x \in \Pi(y)$ implies $x=y$. A basic proximity $\Pi$ on $X$ is called Riesz proximity (RI-proximity) if it satisfies the following additional axiom:

$$
\text { For } x \in X, A, B \in P(X), A, B \in \Pi(x) \text { implies } A \in \Pi(B) \text {. }
$$

2.3. Definition. The operator $c_{\Pi}(A)=[x: x \in \Pi(A)]$ is called the closure operator induced by the proximity $\Pi$.

2.4. THEOREM. For every RI-proximity, $c_{\Pi}$ is a Čech closure operator satisfying the $R_{1}$-axiom.

Proof. The fact that $c_{\Pi}$ is a Čech closure operator is well known. Suppose $y \in c_{\Pi}(x) \cap c_{\Pi}(A)$. Then $y \in \Pi(x)$ and $y \in \Pi(A)$ which, in turn, implies $x \in \Pi(y)$ and $A \in \Pi(y)$. Since $\Pi$ is a Riesz proximity, it follows that $x \in \Pi(A)$.

2.5. THEOREM. Given any $R_{1}$-closure space $(X, c)$, define $\Pi_{0}$ by $A \Pi_{0} B$ iff $c(A) \cap c(B) \neq \varnothing$. Then $\Pi_{0}$ is an $R I$-proximity relation on $X$ and is compatible with the given closure, that is, $c_{\Pi_{0}}=c$.

Proof. That $\Pi_{0}$ is a basic proximity relation on $P(X)$ is a trivial consequence of the closure axioms. To prove that $\Pi_{0}$ is a Riesz proximity, suppose $A, B \in \Pi_{0}(x)$ where $x \in X$. Then $c(A) \cap c(x) \neq \varnothing$ and $c(B) \cap$ $c(x) \neq \varnothing$. Since $c$ is an $R_{1}$-closure, it follows that $x \in c(A) \cap c(B)$ and hence $A \in \Pi_{0}(B)$. Now

$$
\begin{aligned}
c_{\Pi_{0}}(A) & =\left[x \in X: x \in \Pi_{0}(A)\right] \\
& =[x \in X: c(x) \cap c(A) \neq \varnothing]=[x \in X: x \in c(A)]=c(A) .
\end{aligned}
$$

The fact that $c$ is an $R_{1}$-closure has been used to prove the above compatibility.

2.6. TheOREM. Given an RI-proximity space $(X, \Pi)$ and $\Pi_{0}$ defined by $A \Pi_{0} B$ iff $c_{\Pi}(A) \cap c_{\Pi}(B) \neq \varnothing$, we have that $A \Pi_{0} B$ implies $A \Pi B$ for all subsets $A$ and $B$ of $X$. Thus $\Pi_{0}$ is the smallest $R I$-proximity relation compatible with the closure in an $R_{1}$-closure space.

Proof. It follows immediately from 2.4 and 2.5 .

Grills, clans and clusters. Grills were introduced by Choquet [1]. Below we give the definition of a grill. Elementary results on grills are mentioned in Thron [4].

2.7. Definition. A family $\mathcal{G}$ of subsets of $X$ satisfying the properties (i) $B \supset A \in \mathcal{G}$ implies $B \in \mathcal{G}$, (ii) $A \cup B \in \mathcal{G}$ implies $A \in \mathcal{G}$ or $B \in \mathcal{G}$, (iii) $\varnothing \notin \mathcal{G}$, is called a grill. For a fixed $X, \Gamma(X)$ will denote the set of all grills on $X$.

The following facts are evident: (i) For a proper grill $\mathcal{G}$ (nonempty), $A \subset X$ implies $A \in \mathcal{G}$ or $X \backslash A \in \mathcal{G}$. (ii) For a basic proximity space $(X, \Pi), \Pi(A)$ is a grill on $X$ for all $A \in P(X)$. 
2.8. Definitions. For a basic proximity $(X, \Pi)$ a family $\mathcal{G}$ of subsets of $X$ is called a $\Pi$-clan if it satisfies the following conditions: (i) $(i)$ is a grill. (ii) $A, B \in \mathcal{G} \Rightarrow A \in \Pi(B)$.

A $\Pi$-clan $\mathcal{G}$ is said to be a maximal $\Pi$-clan if $\mathcal{G} \subset \mathcal{G}_{1}$, where $\mathcal{G}_{1}$ is another $\Pi$-clan, then $\mathcal{G}=\mathcal{G}_{1}$. A $\Pi$-clan $\mathcal{G}$ is called a $\Pi$-cluster if it satisfies the following additional condition: $\mathcal{G} \subset \Pi(A) \Rightarrow A \in \mathcal{C}_{\text {. }}$.

The following facts are immediate: (a) if $\mathcal{G}_{1}$ and $\mathcal{S}_{2}$ are clusters from $X$ and $\mathcal{G}_{1} \subset \mathcal{G}_{2}$, then $\mathcal{G}_{1}=\mathcal{G}_{2}$. (b) If $A \cap B \neq \varnothing$ for every $B \in \mathcal{G}$, where $\mathcal{G}$ is a cluster, then $A \in \mathcal{G}$. (c) Every $\Pi$-cluster is a maximal $\Pi$-clan.

2.9. Theorem. $A$ basic proximity space $(X, \Pi)$ is an RI-proximity space iff $\Pi(x)$ is a cluster for all $x \in X$.

Proof. Suppose $(X, \Pi)$ is a Riesz proximity space. For $x \in X$, surely $\Pi(x)$ is a grill. Let $A, B \in \Pi(x)$. Since $\Pi$ is a Riesz proximity, it follows that $A \in \Pi(B)$. If $\Pi(x) \subset \Pi(A)$, then $x \in \Pi(A)$ and hence $A \in \Pi(x)$. The converse is an immediate consequence of the definition of the cluster.

2.10. Corollary. If $\mathcal{G}$ is a cluster containing $x$, then $\mathcal{G}=\Pi(x)$.

Proof. The result follows from Definition 2.8 (a) and $\mathcal{G} \subset \Pi(x)$.

3. Main result. To state the main result we shall need the following

3.1. Definition. A subset $Y$ of a closure space $(X, c)$ is regularly dense in $X$ if given $F \subset X, p \notin c(F)$, there exists a subset $E$ of $Y$ with the property $p \in c(E) \subset X-c(F)$.

REMARK. If $Y$ is regularly dense in $X$, then $c(Y)=X$.

3.2. THEOREM. Let $X$ be a set and $\Pi$ a binary relation on $P(X)$. The following are equivalent:

(I) There exists an $R_{1}$-closure space $(Y, c)$ and a mapping $f$ of $X$ into $Y$ such that $f(X)$ is regularly dense in $Y, f$ is a cl-isumorphism of $X$ onto $f(X)$ satisfying $c_{f(X)}(f(x))=f(x)$ and

$$
A \Pi B \text { in } X \text { iff } c(f(A)) \cap c(f(B)) \neq \varnothing .
$$

(II) $\Pi$ is a separated Riesz proximity satisfying the additional axiom:

Given $A \Pi B$ in $X$, there exists a cluster $\mathcal{G}$ to which both $A$ and $B$ belong.

Proof. Suppose (I) holds and define $\Pi$ by (*). That $\Pi$ is a basic proximity follows immediately from the properties of closure. Suppose $x \in \Pi(y)$. Then $c(f(x)) \cap c(f(y)) \neq \varnothing$. Since $c$ is an $R_{1}$-closure, it follows that $f(x) \in$ $c(f(y))$. Thus $f(x) \in c(f(y)) \cap f(X)$ that is, $f(x) \in c_{f(X)}(f(y))=f(y)$. Since $f$ is a cl-isomorphism of $X$ onto $f(X)$, it follows that $x=y$. This proves that $\Pi$ is a separated proximity. We next show that $\Pi$ is a Riesz proximity. For $x \in X, A, B \in P(X)$, suppose $A, B \in \Pi(x)$. Then $c(f(x)) \cap c(f(A)) \neq$ $\varnothing$ and $c(f(x)) \cap c(f(B)) \neq \varnothing$. That the closure operator is $R_{1}$ implies $f(x) \in c(f(A)) \cap c(f(B))$, that is, $A \in \Pi(B)$. It remains to prove for $(A, B)$ $\in \Pi$ there exists a cluster to which both $A$ and $B$ belong. Now $(A, B) \in \Pi$, 
which implies that there exists a $y \in c(f(A)) \cap c(f(B))$. Define

$$
\tau_{y}=[D \subset X: y \in c(f(D))]
$$

Surely $A$ and $B$ are in $\tau_{y}$. We omit the details of the fact that $\tau_{y}$ is a cluster since they are quite similar to the ones given in Lodato [3].

For the converse suppose (II) holds. Given $x \in X$, the class $\Pi(x)$ is a cluster from $X$, by 2.9. For a subset $A$ of $X$, let $A^{*}$ be the set of all clusters to which $A$ belongs. We will denote the set of all clusters from $X$ by $Y$. Observe that

$$
(A \cup B)^{*}=A^{*} \cup B^{*}
$$

since clusters are grills.

Following Lodato [3], we say that a subset $A$ of $X$ absorbs a subset $\beta$ of $Y$ iff $A$ belongs to every cluster in $\beta$, that is, $\beta \subset A^{*}$. For any subset $\beta$ of $Y$, we define $c_{1}(\beta)$ by:

$\mathfrak{B} \in c_{1}(\beta)$ iff every subset $E$ of $X$ which absorbs $\beta$ is in $\mathfrak{B}$.

It follows as in Lodato [3] that

$$
c_{1}\left(\beta_{1} \cup \beta_{2}\right)=c_{1}\left(\beta_{1}\right) \cup c_{1}\left(\beta_{2}\right)
$$

for all subsets $\beta_{1}, \beta_{2}$ in $P(Y)$ and $c_{1}(\mathfrak{B})=\mathfrak{B}$ for every $\mathfrak{B}$ in $Y$.

Let $f$ be the mapping which assigns to each $x$ in $X$ the cluster $\Pi(x)$ determined by it. This mapping is well defined. Define

$$
c(\beta)=\left(f^{-1}(\beta)\right)^{*} \cup c_{1}(\beta) .
$$

Observe that $c(f(A))=A^{*}$. By definition

$$
c(f(A))=\left(f^{-1}(f(A))\right)^{*} \cup c_{1}(f(A))=A^{*} \cup c_{1}(f(A))=A^{*},
$$

since $c_{1}(f(A)) \subset A^{*}$. The inclusion $c_{1}(f(A)) \subset A^{*}$ is a consequence of the fact that $A$ absorbs $f(A)$.

We now show that closure axioms are satisfied by the closure defined by (**).

Since $\beta \subset c_{1}(\beta)$, it follows that $\beta \subset c(\beta)$. The fact that $c(\varnothing)=\varnothing$ is trivial. (3.2.1), (3.2.2) and the fact that $f^{-1}$ distributes on unions imply that $c\left(\beta_{1} \cup \beta_{2}\right)=c\left(\beta_{1}\right) \cup c\left(\beta_{2}\right)$. Thus $(Y, c)$ is a closure space. We shall next show that $(Y, c)$ is an $R_{1}$-closure space. For $\mathscr{B} \in Y, f^{-1}(\mathfrak{B})$ is either empty or equals $x$ for some $x$ in $X$. If $f^{-1}(\mathfrak{B})=\varnothing$, then $c(\mathfrak{B})=c_{1}(\mathfrak{B})=\mathfrak{B}$. On the other hand, if $f^{-1}(\mathfrak{B})=x$ for some $x$ in $X$, then $\mathfrak{B}=\Pi(x)$. Hence

$$
c(\mathfrak{B})=\left(f^{-1}(\mathfrak{B})\right)^{*} \cup c_{1}(\mathfrak{B})=\Pi(x) \cup \Pi(x)=\Pi(x)=\mathfrak{B} .
$$

The separated character of Riesz proximity implies $f$ is one-one. That $f$ is a cl-isomorphism shall be accomplished by showing (i) $c_{f(X)}(f(A)) \supset f\left(c_{\Pi}(A)\right)$ for every $A$ in $P(X)$, and (ii) $f^{-1}\left(c_{f(X)}(f(A))\right) \subset c_{\Pi}(A)$ for each $A \subset X$. For (i), suppose $x \in c_{\Pi}(\dot{A})$. Then $A \in \Pi(x)$. Thus $\Pi(x) \in A^{*}=c(f(A))$ which, in turn, implies $\Pi(x) \in c_{f(X)}(f(A))$. In order to prove (ii), suppose $\mathfrak{B} \in$ $c_{f(X)}(f(A))$. Then there exists an $x \in X$ such that $\mathfrak{B}=\Pi(x)$ and $\Pi(x) \in$ 
$c_{f(X)}(f(A))=c(f(A)) \cap f(X)$. Thus $A \in \Pi(x)$, that is, $x \in c_{\Pi}(A)$.

$A \Pi B$ iff there exists a cluster to which both $A$ and $B$ belong, that is, $A^{*} \cap B^{*} \neq \varnothing$; thus $c(f(A)) \cap c(f(B)) \neq \varnothing$ iff $A \Pi B$.

It remains to check that $f(X)$ is regularly dense in $Y$. Suppose $\beta \subset Y$ and $\mathfrak{B}_{0} \notin c(\beta)=\left(f^{-1}(\beta)\right)^{*} \cup c_{1}(\beta)$. Then $f^{-1}(\beta) \notin \mathfrak{B}_{0}$ and there exists a subset $A$ which absorbs $\beta$ and does not belong to $\mathfrak{B}_{0}$. Since $\mathfrak{B}_{0}$ is, in particular, a grill, it follows that $A \cup f^{-1}(\beta) \notin \mathfrak{B}_{0}$. Using the fact that $\mathscr{B}_{0}$ is a cluster, it follows that there exists a $B \in \mathfrak{B}_{0}$ such that $A \cup f^{-1}(\beta) \notin \Pi(B)$, that is, $A \notin \Pi(B)$ and $f^{-1}(\beta) \notin \Pi(B)$. Let $B$ be any element of $B^{*}$. Then $B \in \mathfrak{B}$ and hence $f^{-1}(\beta)$ and $A$ do not belong to $\mathscr{B}$. Thus it follows that $\mathfrak{B} \in$ $Y \backslash c(\beta)$. Clearly $\mathfrak{B}_{0} \in B^{*}=c(f(B)) \subset Y \backslash c(\beta)$. This completes the proof.

We end this section with an example of a Riesz proximity space in which a pair of proximal sets are contained in no cluster.

3.3. Example. Let $X=X_{1} \cup X_{2}, X_{1} \cap X_{2}=\varnothing$ and $X_{1}$ and $X_{2}$ are both infinite. Define a closure $c$ on $X$ by

$c(D)= \begin{cases}D & \text { if } D \text { is a finite subset of } X \\ X_{i} \cup D & \text { if } D \text { is infinite and } X_{j} \cap D \text { is finite, } i, j=1,2 \text {, and } i \neq j, \\ X & \text { otherwise. }\end{cases}$

$(X, c)$ is an $R_{1}$-closure space. In fact, it is a $T_{1}$-topological space. We next define a binary relation $\Pi$ on $P(X)$ as follows: $(D, E) \in \Pi$ iff $c(D) \cap c(E)$ $\neq \varnothing$ or both $D$ and $E$ are infinite. $(X, \Pi)$ is a Riesz proximity space. Moreover,

$$
\Pi(x)=\left[D: x \in D \text { or } X_{i} \cap D \text { is infinite }\right]
$$

if $x \in X_{i}, i=1$, 2. That $\Pi(x)$ is a cluster follows from the fact that $(X, \Pi)$ is a Riesz proximity space. Consider

$$
\mathcal{G}^{*}=[D: D \text { is an infinite subset of } X] .
$$

$\mathcal{G}^{*}$ is a maximal $\Pi$-clan. For $x_{i} \in X_{i}, i=1,2, \mathcal{G} * \subset \Pi\left(\left[x_{1}, x_{2}\right]\right)$. However, $\left[x_{1}, x_{2}\right] \notin \mathcal{G} *$. Thus $\mathcal{G} *$ is not a cluster. Let $\mathfrak{B}$ be any $\Pi$-cluster. Then $\mathfrak{B} \not \subset \mathcal{G}^{*}$, for otherwise $\mathfrak{B}=\mathcal{G}^{*}$-a contradiction. Thus there exists an $x \in X$ such that $[x] \in \mathfrak{B}$ and this implies that $\mathfrak{B}=\Pi(x)$. Clearly $\left(X_{1}, X_{2}\right) \in \Pi$, but there exists no $\Pi(x)$ to which both $X_{1}$ and $X_{2}$ belong, for the existence of such an $x$ would contradict the fact that $X_{1} \cap X_{2}=\varnothing$.

ACKNOWLEDGEMENT. The authors would like to thank the referee for useful comments.

\section{REFERENCES}

1. G. Choquet, Sur les notions de filtre et de grille, C. R. Acad. Sci. Paris 224 (1947), 171-173. MR 8, 333. 
2. V. A. Efromovič, The geometry of proximity. I, Mat. Sb. 31 (73) (1952), 189-200. MR 14, 1106.

3. M. W. Lodato, On topologically induced generalised proximity relations, Proc. Amer. Math. Soc. 15 (1964), 417-422. MR 28 \#4513

4. W. J. Thron, Proximity structures and grills, Math. Ann. 206 (1973), 35-62. MR 49 \# 1483.

5. Yu. M. Smirnov, On proximity spaces, Mat. Sb. 31 (73) (1952), 543-574; English transl., Amer. Math. Soc. Transl. (2) 38 (1964), 5-35. MR 14, 1107.

Department of Mathematics, Punjab University, Chandigarh-160014, India 